\title{
Early outcome and blood-brain barrier integrity after co-administered thrombolysis and hyperbaric oxygenation in experimental stroke
}

\author{
Dominik Michalski ${ }^{1 *}$, Johann Pelz ${ }^{1,2}$, Christopher Weise ${ }^{1,2}$, Johannes Kacza ${ }^{3}$, Johannes Boltze ${ }^{4,5}$, Jens Grosche ${ }^{2}$, \\ Manja Kamprad ${ }^{6}$, Dietmar Schneider ${ }^{1}$, Carsten Hobohm ${ }^{1}$ and Wolfgang Härtig ${ }^{2}$
}

\begin{abstract}
Background: After promising results in experimental stroke, normobaric (NBO) or hyperbaric oxygenation ( $\mathrm{HBO}$ ) have recently been discussed as co-medication with tissue plasminogen activator (tPA) for improving outcome. This study assessed the interactions of hyperoxia and tPA, focusing on survival, early functional outcome and blood-brain barrier (BBB) integrity following experimental stroke.

Methods: Rats $(n=109)$ underwent embolic middle cerebral artery occlusion or sham surgery. Animals were assigned to: Control, NBO (60-minute pure oxygen), HBO (60-minute pure oxygen at 2.4 absolute atmospheres), tPA, or HBO+tPA. Functional impairment was assessed at 4 and 24 hours using Menzies score, followed by intravenous application of FITC-albumin as a BBB permeability marker, which was allowed to circulate for 1 hour. Further, blood sampling was performed at 5 and 25 hours for MMP-2, MMP-9, TIMP-1 and TIMP-2 concentration.

Results: Mortality rates did not differ significantly between groups, whereas functional improvement was found for $\mathrm{NBO}, \mathrm{tPA}$ and $\mathrm{HBO}+\mathrm{tPA}$. NBO and $\mathrm{HBO}$ tended to stabilize BBB and to reduce MMP-2. tPA tended to increase BBB permeability with corresponding MMP and TIMP elevation. Co-administered HBO failed to attenuate these early deleterious effects, independent of functional improvement.

Conclusions: The long-term consequences of simultaneously applied tPA and both NBO and HBO need to be addressed by further studies to identify therapeutic potencies in acute stroke, and to avoid unfavorable courses following combined treatment.
\end{abstract}

\section{Background}

The repeated translational failures of preclinical approaches in acute ischemic stroke necessitate a more complex perspective of tissue salvaging and regeneration in experimental research [1], involving functional keystructures such as the blood-brain barrier (BBB) with the associated 'neurovascular unit' [2-5]. Thromboembolic models are considered to provide best comparability to the human pathophysiology, thus being of increasing interest $[5,6]$. Along with the growing knowledge on timedependent BBB changes $[7,8]$, matrix metalloproteinases (MMPs) and their inhibitors (TIMPs) were proposed as

\footnotetext{
* Correspondence: dominik.michalski@medizin.uni-leipzig.de 'Department of Neurology, University of Leipzig, Liebigstr. 20, 04103 Leipzig, Germany

Full list of author information is available at the end of the article
}

mediators with deleterious effects in early phases, but also regenerative properties in subsequent phases $[9,10]$. The application of oxygen under normobaric (NBO) or hyperbaric (HBO) conditions was shown to be beneficial in experimental studies by decreasing infarct size, functional impairment, hemorrhagic transformation, and early MMP response leading to BBB stabilization [11-17]. Recently, hyperoxia was discussed as co-medication to tissue plasminogen activator (tPA) $[17,18]$, which even results in clinical improvement [19], but is also known for MMP activation and BBB breakdown with possible hemorrhagic transformation [20-22]. Hence, hyperoxia might attenuate deleterious tPA effects and improve the clinical outcome [18]. Our group previously found that simultaneous treatment with tPA and HBO may have detrimental effects on long-term outcome in rats, possibly by influencing $\mathrm{BBB}$ 
integrity due to early interactions [23]. The present study aimed on interacting effects of hyperoxia and tPA with special emphasis on survival, early functional outcome, $\mathrm{BBB}$ integrity and related mediators in a translational relevant setting after thromboembolic stroke in rats.

\section{Methods}

\section{Experimental design and interventions}

The experimental protocol involving animals was approved by local authorities [Regierungspräsidium Leipzig, Tierversuchsvorhaben (TVV) 02/09] and has been conducted according to the European Communities Council Directive (86/609/EEC). Male Wistar rats (mean weight $316.2 \mathrm{~g}$ ), provided by Charles River (Sulzfeld, Germany), underwent right-sided middle cerebral artery occlusion (MCAO; $\mathrm{n}=105)$ or sham operation $(\mathrm{n}=4)$, as described below. Thirteen animals died during surgery and 4 animals were excluded from the study due to minor functional impairment (Menzies score 0-1) indicating insufficient cerebral ischemia, as specified previously. Finally, 92 animals were consecutively assigned to sham surgery $(\mathrm{n}=4)$, MCAO control $(\mathrm{n}=17)$, NBO $(\mathrm{n}=18), \mathrm{HBO}(\mathrm{n}=16), \mathrm{tPA}(\mathrm{n}=19)$, or HBO+tPA $(\mathrm{n}=$ 18). Treatment was initiated 2 hours after ischemia onset: The NBO group received 60 minutes of normobaric pure oxygen in a hyperbaric chamber (Sayers/Hebold, Cuxhaven, Germany), the HBO group underwent 60 minutes (plus compression/decompression) of $100 \% \mathrm{O}_{2}$ at 2.4 absolute atmospheres (ATA). tPA-treated animals received $9 \mathrm{mg} / \mathrm{kg}$ bodyweight $\mathrm{tPA}$ (Actilyse, Boehringer, Ingelheim, Germany) intravenously over 30 minutes. The $\mathrm{HBO}+\mathrm{tPA}$ group simultaneously received tPA and $\mathrm{HBO}$ beginning at 2 hours. Functional impairment was assessed after surgery (baseline) and 4- or rather 24-hour observation period post MCAO, iimmediately followed by fluorescein isothiocyanate (FITC)-albumin injection via a femoral vein $(20 \mathrm{mg} / 1 \mathrm{~mL}$ saline; Sigma, Taufkirchen, Germany) for visualization of BBB permeability. After usually 1 hour of circulation, animals were sacrificed in deep narcosis. Blood samples were drawn transcardially, followed by perfusion with saline and $4 \%$ paraformaldehyde in phosphate-buffered saline (PFA). Brains were removed from skull, immersed in the same fixative for 24 hours and equilibrated in 30\% buffered sucrose. All study endpoints were defined prior to the first animal enrollment.

\section{Surgical procedure}

MCAO was induced using an embolic model according to Zhang et al. [24] with minor modifications as described previously [23]. Briefly, a polyethylene (PE) tubing with tapered end was advanced from an incision of the external carotid artery (ECA) into the internal carotid artery, reaching the origin of the middle cerebral artery. A weight-adapted blood clot (mean length 46 $\mathrm{mm}$ ) was injected, followed by catheter removal and ligation of the ECA stump. The sham operation was performed in the same manner, including preparation of cervical vessels, but without catheter insertion. In all groups, PE tubes were inserted into the femoral vein for tPA administration and the femoral artery for monitoring heart rate, arterial pressure (Datex, Helsinki, Finland), and obtaining blood samples $\left(\mathrm{PaO}_{2}\right.$ and glucose; ABL 700, Radiometer, Copenhagen, Denmark) during surgery and after therapy. Animals were anesthetized using $2-2.5 \%$ isoflurane $\left(70 \% \mathrm{~N}_{2} \mathrm{O} / 30 \% \mathrm{O}_{2}\right)$ during surgery. Thereby, the body temperature was adjusted to $37.0^{\circ} \mathrm{C}$ using a thermostatically controlled heating pad (Fine Science Tools, Heidelberg, Germany) with rectal probe.

\section{Assessment on mortality and functional impairment}

The rate of premature death was recorded in each treatment group during the observation period including associated FITC-albumin injection. The Menzies score was used to assess functional impairment [25], scaled from 0 (no apparent deficits) up to 4 (spontaneous contralateral circling).

\section{Tissue preparation, imaging and quantification of BBB permeability}

The visualization technique of BBB impairment after in vivo application of FITC-albumin as permeability marker was described previously [26]. Briefly, $30 \mu \mathrm{m}$-thick serial brain sections were cut coronally using a freezing microtome. Ten series of sections per animal were stored in $0.1 \mathrm{~mol} / \mathrm{L}$ Tris-buffered saline (TBS; $\mathrm{pH}$ 7.4) containing sodium azide. After washing, sections were mounted on fluorescence-free slides, air-dried and coverslipped with Entellan in toluene (Merck, Darmstadt, Germany). Subsequent to a screening microscopy (Axioplan, Zeiss, Jena, Germany) for identification of sections with the clearest infarct presentation by FITC-albumin leakage, the neighboring serial sections were blocked (TBS, $2 \%$ bovine serum albumin, $0.3 \%$ Triton X-100 [TBS-BSA-T]), followed by an enhancement of the green fluorescent signal with carbocyanine (Cy)2-anti-FITC IgG $(20 \mu \mathrm{g} / \mathrm{mL}$ TBSBSA-T; Jackson ImmunoResearch, West Grove, PA) for long-term stabilization. Moreover, vascular membranes were detected by SMI-71 (1:400; Emeryville, CA [27]) and Cy3-donkey-anti-mouse IgM $(20 \mu \mathrm{g} / \mathrm{mL}$ TBS-BSA; Jackson), colored in red. Finally, washing, mounting and coverslipping were performed as described above. Fluorescence-based paravasal intensity of enhanced FITCalbumin served as surrogate for BBB permeability, as exemplarily shown in Figure 1, and was analyzed by a blinded investigator. For this purpose, a fluorescence microscope (Axioplan 2, Zeiss) with structured-light 

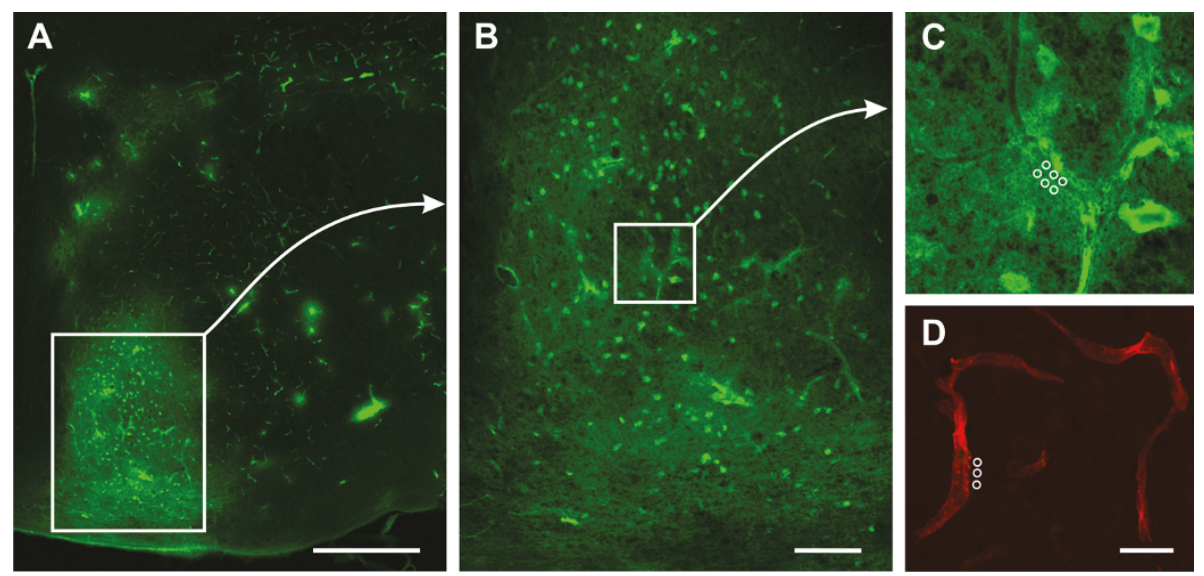

Figure 1 Demonstration of enhanced FITC-albumin measurement as BBB leakage marker at 5 and 25 hours after embolic stroke in rats by confocal laser scanning micrographs. (A), representative striatal ischemia-related leakage of Cy2-enhanced FITC-albumin at $5 \times$ magnification in a NBO-treated animal at 5 hours. (B), enhanced FITC-albumin in ischemic border zone at 10x magnification showing impaired vascular structures with leakage phenomena. (C), para- and intravasal inserted region of interests at $63 \times$ magnification. (D), $63 \times$ magnification of the corresponding area at the non-ischemic hemisphere: Detection of FITC-albumin background staining combined with Cy3-immunolabeling of SMI-71 for vessel localization. Absent leakage indicates BBB integrity. Scale bars: in A $=500 \mu \mathrm{m}$, in $B=100 \mu \mathrm{m}$, and in $\mathrm{D}=25 \mu \mathrm{m}$.

confocal system (OptiGrid, Qioptiq, Fairport, NY), digital camera (ORCA-ER, Hamamatsu, Hamamatsu City, Japan) and imaging software (Volocity 4.3, PerkinElmer, Waltham, MA) was used. Routinely, 3 vessels in the lesion-bordering zone with clearest leakage phenomena were selected. Six measurements were performed for each of the chosen vessels by using cylindrical region of interests (ROIs; diameter $2 \mu \mathrm{m}$ ) with 9 confocal layers (zinterval $0.5 \mu \mathrm{m}$ ) at each point, obtained at $40 \mathrm{x}$ magnification using a water immersion objective (numerical aperture 1.4), with further digital zoom if necessary. ROIs were positioned intravasally $(\mathrm{n}=3)$ and with distance of approximately $2 \mu \mathrm{m}$ from the vessel wall $(n=3)$. To control for any background staining, 1 corresponding vessel in the contra-lesional hemisphere was identified by SMI$71 / \mathrm{Cy} 3$ and paravasal space was measured by 3 ROIs in the same manner. For all measurements the following setup was applied: Exposure time $80 \mathrm{~ms}$, gain 0, offset 0, super grid quality, grid gain 3x, excitation Cy2 $480 \mathrm{~nm}$, emission $527 \mathrm{~nm}$, excitation Cy3 $545 \mathrm{~nm}$, emission 610 $\mathrm{nm}$. The obtained mean intensity within the ROIs was used for further processing. Finally, means of 3 ipsilesional vessels and 1 contra-lesional vessel were calculated and subtracted for total leakage. Furthermore, a surrogate for BBB integrity was calculated as extra-/intravasal ratio on the affected hemisphere indicating the leakage proportion. For optimal presentation, micrographs of fluorescence labeling were obtained using a 510 Meta laser scanning microscope (Zeiss), equipped with an argon laser $(488 \mathrm{~nm}$ ) for the excitation of $\mathrm{Cy} 2$, and two helium-neon lasers for the excitation of Cy 3 (543 $\mathrm{nm})$. For the detection of emitted spectra, the following band-pass filters were used: 500-530 nm for Cy2 (green), and $565-615 \mathrm{~nm}$ for Cy3 (red). The original images were processed with Adobe Photoshop 7.0 (Adobe Systems Inc., Mountain View, CA). Thereby, brightness, contrast and sharpness of the final pictures were slightly modified.

\section{Measuring BBB-related serum markers}

Samples were allowed to clot, and then centrifuged and finally stored up to $-80^{\circ} \mathrm{C}$. After thawing, MMP-2, MMP-9, TIMP-1 and TIMP-2 concentrations were determined in a blinded fashion by using enzyme-linked immunosorbent assays (USCN, Wuhan, China; E90100Ra/E90553Ra/E90552Ra/E90128Ra). The minimum detectable dose, which was differentiable from zero (as listed in the instruction manual), was utilized for values under the minimum or with uncertain calculation at the lower standard curve.

\section{Statistical analysis}

All calculations were performed with SPSS Vers. 18.0 (SPSS Inc, an IBM Company, Chicago, IL). One-way analysis of variance (ANOVA) was applied for intergroup differences at a single time point, followed by the Duncan test considering multiple means. For intragroup testing at different time points, WilcoxonMann-Whitney and Kolmogorov-Smirnov tests were performed. Percentage rates were verified using the Chi-square test with additional Monte Carlo simulation (confidence interval $=99 \%$, samples $=10,000$ ), ensuring exact testing. Pearson correlations were applied for interrelations. A $P<0.05$ was considered as statistically significant. 


\section{Results}

Physiological parameters were similar between groups with exception of weight (slightly increased in shamand tPA-treated animals), and marginal differences in temperature, mean arterial pressure and $\mathrm{PaO}_{2}$ - overall indicating no major group differences (Table 1). As expected for this model, ischemia predominantly occurred in the proximal section of the middle cerebral artery territory with varying infarct sizes as indicated by FITC-albumin leakage.

Mortality and early functional impairment

Premature death occurred in a total of 8 animals $(8.7 \%)$. The distribution according to the treatment groups is shown in Figure 2A, and was not statistically significant (Chi-square, $P=0.617$; Monte Carlo, $P=$ 0.678). As expected, no functional impairment was found in sham-assigned animals. Figure $2 \mathrm{~B}$ indicates the course of functional impairment depending on treatment. At baseline and 4 hours, impairment did not differ between the groups (ANOVA, $P=0.073$, $P=0.437)$; at 24 hours differences were found (ANOVA, $P=0.004$ ) with superiority for NBO, tPA and $\mathrm{HBO}+\mathrm{tPA}$ compared with control and HBO (Duncan, $P<0.05)$. Regarding the time course, controls showed a progressive functional impairment during the first 24 hours (Wilcoxon-Mann-Whitney, $P=0.044$ ), whereas improvement was noted for NBO, tPA and

Table 1 Physiological parameters

\begin{tabular}{|c|c|c|c|c|c|c|c|}
\hline & $\begin{array}{c}\text { Sham } \\
\text { mean } \pm \text { SD }\end{array}$ & $\begin{array}{c}\text { Control } \\
\text { mean } \pm \text { SD }\end{array}$ & $\begin{array}{c}\mathrm{NBO} \\
\text { mean } \pm \text { SD }\end{array}$ & $\begin{array}{c}\mathrm{HBO} \\
\text { mean } \pm \mathrm{SD}\end{array}$ & $\begin{array}{c}\mathrm{tPA} \\
\text { mean } \pm \mathrm{SD}\end{array}$ & $\begin{array}{c}\mathrm{HBO}+\mathrm{tPA} \\
\text { mean } \pm \mathrm{SD}\end{array}$ & $\begin{array}{c}\text { ANOVA } \\
\mathrm{F}(P)\end{array}$ \\
\hline Weight (g) & $357.8 \pm 10.9$ & $324.1 \pm 45.6$ & $304.3 \pm 26.2$ & $296.9 \pm 26.5$ & $334.8 \pm 36.6$ & $317.0 \pm 17.5$ & $4.6\left({ }^{(*)}\right) \dagger$ \\
\hline Duration of surgery (minutes) & $63.8 \pm 9.1$ & $74.5 \pm 25.5$ & $66.6 \pm 18.2$ & $71.0 \pm 22.8$ & $68.3 \pm 14.7$ & $58.1 \pm 12.6$ & 1.5 (n.s.) \\
\hline \multicolumn{8}{|l|}{ Temperature $\left({ }^{\circ} \mathrm{C}\right)$} \\
\hline Initial & $37.5 \pm 0.4$ & $37.5 \pm 0.4$ & $37.6 \pm 0.5$ & $37.7 \pm 0.3$ & $37.5 \pm 0.3$ & $37.6 \pm 0.5$ & 0.7 (n.s.) \\
\hline After catheter & $37.6 \pm 0.2$ & $37.3 \pm 0.3$ & $37.2 \pm 0.3$ & $37.1 \pm 0.4$ & $37.2 \pm 0.7$ & $37.2 \pm 0.4$ & 0.9 (n.s.) \\
\hline After clot injection & $37.5 \pm 0.0$ & $37.4 \pm 0.2$ & $37.3 \pm 0.3$ & $37.2 \pm 0.3$ & $37.3 \pm 0.7$ & $37.1 \pm 0.6$ & 1.1 (n.s.) \\
\hline End of surgery & $37.6 \pm 0.4$ & $37.5 \pm 0.3$ & $37.2 \pm 0.2$ & $37.3 \pm 0.3$ & $37.4 \pm 0.4$ & $37.0 \pm 0.5$ & $3.6(* *) \neq$ \\
\hline After therapy & $\mathrm{n} / \mathrm{a}$ & $\mathrm{n} / \mathrm{a}$ & $36.8 \pm 0.8$ & $37.3 \pm 0.7$ & $37.2 \pm 0.8$ & $36.9 \pm 0.8$ & 1.7 (n.s.) \\
\hline \multicolumn{8}{|l|}{ Mean arterial pressure $(\mathrm{mmHg})$} \\
\hline After catheter & $75.7 \pm 3.5$ & $82.9 \pm 6.5$ & $78.1 \pm 8.4$ & $77.8 \pm 8.4$ & $74.4 \pm 9.0$ & $79.9 \pm 8.2$ & 2.2 (n.s.) \\
\hline After clot injection & $72.5 \pm 3.5$ & $78.2 \pm 6.3$ & $73.3 \pm 5.8$ & $77.1 \pm 9.0$ & $72.4 \pm 7.9$ & $77.7 \pm 11.8$ & 1.5 (n.s.) \\
\hline End of surgery & $62.0 \pm 3.0$ & $75.3 \pm 8.3$ & $74.8 \pm 13.2$ & $79.1 \pm 11.7$ & $71.4 \pm 7.2$ & $81.9 \pm 12.6$ & $3.0\left(^{*}\right) \S$ \\
\hline After therapy & $\mathrm{n} / \mathrm{a}$ & $\mathrm{n} / \mathrm{a}$ & $96.0 \pm 15.5$ & $86.9 \pm 10.7$ & $84.4 \pm 12.9$ & $86.9 \pm 17.0$ & 2.1 (n.s.) \\
\hline \multicolumn{8}{|l|}{ Heart rate (per minute) } \\
\hline After catheter & $206.7 \pm 42.5$ & $188.5 \pm 17.5$ & $189.3 \pm 12.6$ & $191.5 \pm 21.8$ & $197.0 \pm 18.5$ & $192.7 \pm 26.9$ & 0.7 (n.s.) \\
\hline After clot injection & $220.0 \pm 49.5$ & $194.5 \pm 12.0$ & $187.9 \pm 17.0$ & $203.1 \pm 27.7$ & $192.4 \pm 21.7$ & $203.9 \pm 23.2$ & 1.8 (n.s.) \\
\hline End of surgery & $220.0 \pm 40.9$ & $198.5 \pm 12.3$ & $200.6 \pm 25.3$ & $200.9 \pm 22.2$ & $192.8 \pm 18.9$ & $200.2 \pm 19.0$ & 1.0 (n.s.) \\
\hline After therapy & $\mathrm{n} / \mathrm{a}$ & $\mathrm{n} / \mathrm{a}$ & $203.1 \pm 13.3$ & $194.5 \pm 13.0$ & $204.2 \pm 18.0$ & $212.2 \pm 21.7$ & 2.7 (n.s.) \\
\hline \multicolumn{8}{|l|}{$\mathrm{PaO}_{2}(\mathrm{mmHg})$} \\
\hline After catheter & $97.4 \pm 9.1$ & $102.8 \pm 13.7$ & $104.2 \pm 22.6$ & $97.9 \pm 14.5$ & $98.6 \pm 9.2$ & $105.1 \pm 12.9$ & 0.7 (n.s.) \\
\hline End of surgery & $105.3 \pm 12.3$ & $114.1 \pm 29.5$ & $118.6 \pm 19.9$ & $108.5 \pm 18.3$ & $107.6 \pm 13.8$ & $114.7 \pm 14.5$ & 0.8 (n.s.) \\
\hline After therapy & $\mathrm{n} / \mathrm{a}$ & $\mathrm{n} / \mathrm{a}$ & $117.8 \pm 23.9$ & $118.4 \pm 18.2$ & $117.7 \pm 13.6$ & $149.8 \pm 28.9$ & $8.8\left(^{(* *)} \|\right.$ \\
\hline \multicolumn{8}{|l|}{ Blood glucose $(\mathrm{mmo} / \mathrm{l})$} \\
\hline After catheter & $13.5 \pm 1.7$ & $10.5 \pm 1.9$ & $12.2 \pm 2.3$ & $11.8 \pm 1.5$ & $11.1 \pm 2.5$ & $11.4 \pm 1.6$ & 2.1 (n.s.) \\
\hline End of surgery & $13.2 \pm 1.5$ & $10.4 \pm 1.9$ & $11.8 \pm 3.3$ & $10.6 \pm 1.6$ & $11.2 \pm 3.1$ & $11.6 \pm 2.4$ & 0.9 (n.s.) \\
\hline After therapy & $\mathrm{n} / \mathrm{a}$ & $\mathrm{n} / \mathrm{a}$ & $9.2 \pm 1.8$ & $10.0 \pm 1.8$ & $8.8 \pm 1.4$ & $9.9 \pm 1.5$ & 2.2 (n.s.) \\
\hline
\end{tabular}

$P:{ }^{*}<0.05,{ }^{* *}<0.01,{ }^{* * *}<0.001 ;$ Duncan test: $\dagger$, sham/tPA $>$ control/NBO/HBO/HBO+tPA; $\neq, \mathrm{HBO}+\mathrm{tPA}<$ sham/control/HBO/tPA; $\S$, sham $<$ control/NBO/HBO/tPA/HBO $+\mathrm{tPA} ; \mathrm{II}, \mathrm{HBO}+\mathrm{tPA}>\mathrm{NBO} / \mathrm{HBO} / \mathrm{tPA}$ 


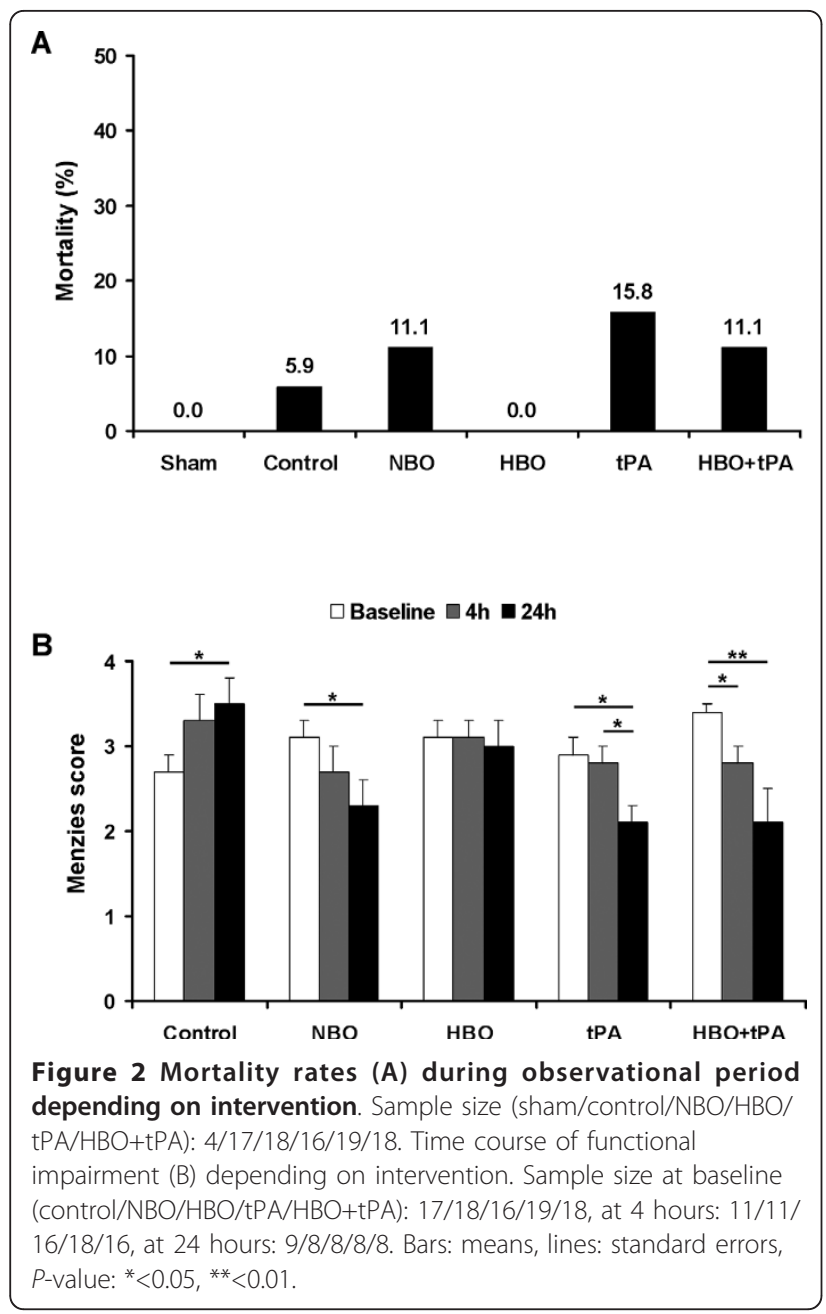

$\mathrm{HBO}+\mathrm{tPA}$ (Wilcoxon-Mann-Whitney, NBO-baseline/ 24 hours $P=0.044$, tPA-baseline $/ 24$ hours $P=0.023$, $4 / 24$ hours $P=0.039, \mathrm{HBO}+\mathrm{tPA}-$ baseline $/ 4$ hours $P=$ 0.006 , baseline/24 hours $P=0.048)$. No time-dependent changes appeared in HBO-treated animals.

\section{BBB permeability}

FITC-albumin leakage was not observed in sham animals (data not shown). In controls, total leakage declined from 5 to 25 hours (Figure 3A). Concerning the treatment, total leakage differed at 5 hours (ANOVA, $P=0.046$ ) between tPA and NBO, as well as between tPA and HBO + tPA (Duncan, $P<0.05$ each). HBO tended to reduce leakage, but this was not statistically significant. At 25 hours, no group-specific differences of total leakage occurred (ANOVA, $P=0.413$ ), whereas combined treatment with $\mathrm{HBO}$ and $\mathrm{TPA}$ tended to increased leakage. Concerning the time course, controls and NBO-treated animals showed a significant reduction from initial values, indicating dynamic properties of BBB integrity (Kolmogorov-Smirnov, $P=0.022, P=0.004$ ).
Extra-/intravasal ratios (Figure 3B) did not differ between treatment groups at either 5 or 25 hours (ANOVA, $P=0.097, P=0.682$ ). At 25 hours, oxygen application (NBO or $\mathrm{HBO}$ ) tended to have the lowest leakage proportions, in contrast to worsened tPA- and $\mathrm{HBO}+\mathrm{tPA}$-treated animals. Time-dependent changes were solely found for NBO with a decreasing value (Kolmogorov-Smirnov, $P=0.022$ ). The leakage proportion of the whole sample significantly correlated with functional impairment at baseline and 4 hours (Pearson, $r=0.391$, $P=0.000, r=0.392, P=0.001)$, but failed to correlate at 24 hours (Pearson, $r=0.186, P=0.251$ ).

\section{BBB-related serum markers}

As shown in Figure 4, significantly lower MMP-2 levels were detected in sham animals at 5 hours as compared to all other groups (ANOVA, $P=0.012$; Duncan, $P<0.05$ ). At 25 hours, $\mathrm{HBO}$ resulted in decreased MMP-2 levels as compared to tPA and HBO+tPA (ANOVA, $P=0.001$; Duncan, $P<0.05)$. Concerning the time course, MMP-2 levels decreased in controls, and NBO-, HBO- and tPAtreated animals (Kolmogorov-Smirnov, $P=0.001, P=$ $0.001, P=0.001, P=0.037$ ). As shown in Figure $4 \mathrm{~B}$, no significant treatment-related differences occurred for MMP-9 (ANOVA, 5 hours $P=0.316,25$ hours $P=$ 0.554 ), but decreasing levels between 5 and 25 hours were found in controls, $\mathrm{HBO}$ - and $\mathrm{HBO}+\mathrm{tPA}$-treated animals (Kolmogorov-Smirnov, $P=0.004, P=0.030, P=$ 0.004). For TIMP-1 (Figure 4C), tPA and $\mathrm{HBO}+\mathrm{tPA}-$ assigned animals revealed increased levels at 5 hours compared to sham, control and NBO (ANOVA, $P=$ 0.000; Duncan, $P<0.05)$. At 25 hours, $\mathrm{HBO}+\mathrm{tPA}$ showed the highest TIMP-1 levels, followed by tPA/sham and HBO (ANOVA, $P=0.000$; Duncan, $P<0.05$ ). For $\mathrm{HBO}$, the TIMP-1 levels significantly declined between 5 and 25 hours (Kolmogorov-Smirnov, $P=0.037$ ). TIMP-2 (Figure 4D) demonstrated no treatment-specific differences at 5 hours (ANOVA, $P=0.388$ ), but the co-administration of $\mathrm{HBO}$ and $\mathrm{TPA}$ led to increased TIMP-2 levels at 25 hours as compared to $\mathrm{HBO}$, control and sham (ANOVA, $P=0.011$; Duncan, $P<0.05$ ). A TIMP-2 reduction between 5 and 25 hours was noted for controls, HBO- and tPA-treated animals (KolmogorovSmirnov, $P=0.001, P=0.001, P=0.007)$.

\section{Discussion}

For the first time, the present data provide insights into complex BBB processes following hyperoxia, supplemented by testing $\mathrm{HBO}$ and $\mathrm{PAA}$ simultaneously. This study focused on changes of $\mathrm{BBB}$ integrity in the early stages following stroke due to their potential long-term relevance. In consideration of translational aspects [5,6], an embolic model was chosen and treatment initiated after ischemia onset without preconditioning attempts. 

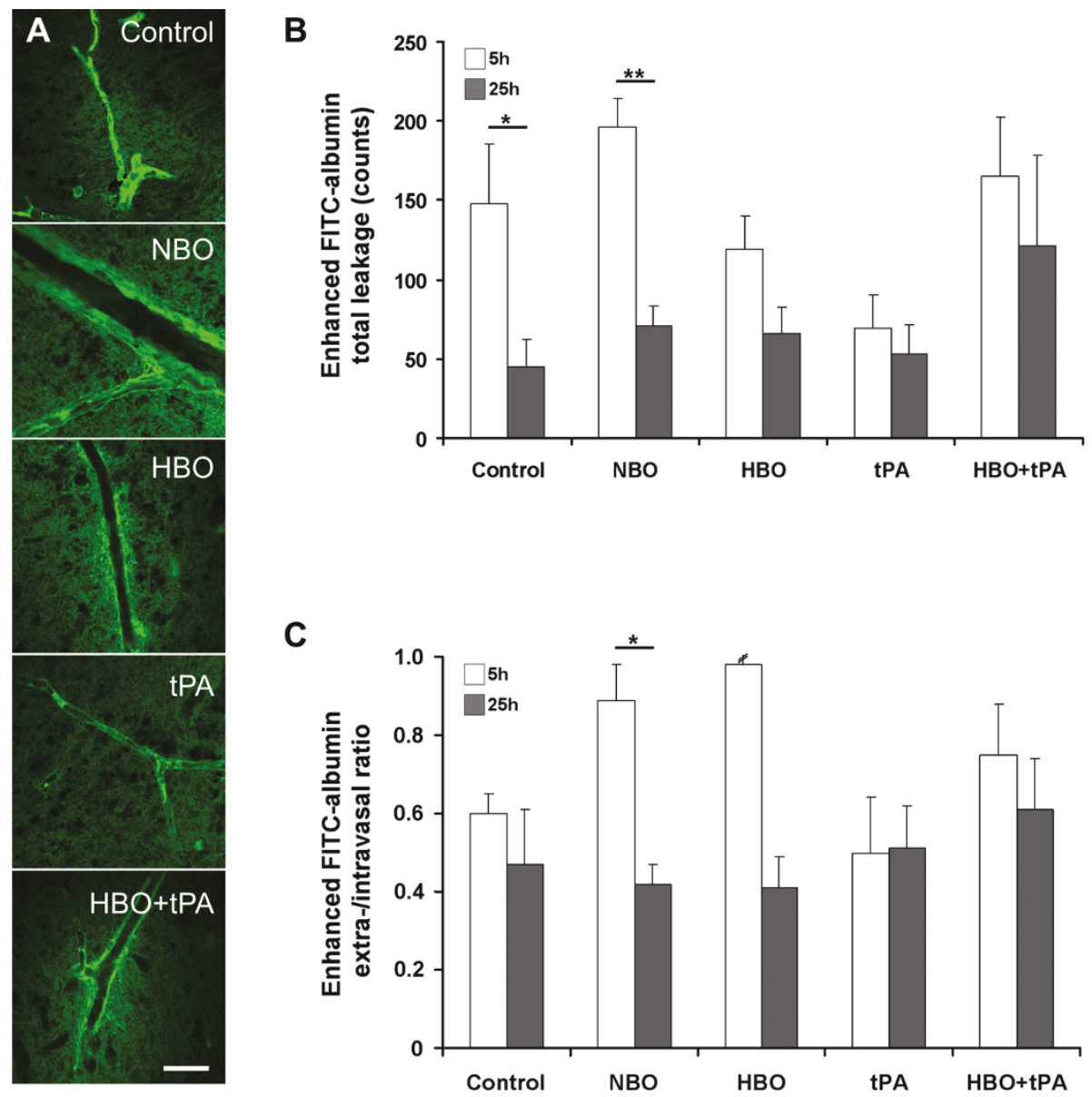

Figure 3 Treatment-dependent representative samples of impaired vessels (A) at 5 hours displayed by confocal laser scanning micrographs (100x magnification; scale bar $=\mathbf{2 0} \boldsymbol{\mu m}$ ). Total paravasal leakage (B) of FITC-albumin on ischemic hemisphere considering background staining as detected on contra-lesional hemisphere and extra-/intravasal ratio (C), calculated by means on affected hemisphere. Sample size for leakage and ratio at 5 hours (control/NBO/HBO/tPA/HBO+tPA): 8/8/8/7/8, at 25 hours: 8/8/8/8/8. Bars: means, lines: standard errors, $P$-value: ${ }^{*}<0.05,{ }^{* *}<0.01$.

The mortality rates did not differ significantly between groups, notably for the combined treatment with $\mathrm{HBO}$ and $\mathrm{TPA}$. This finding reinforced previous reports that focused on additional NBO to tPA and provided comparable results when applied simultaneously [28], or consecutively by starting with NBO [12,29]. Functional impairment deteriorated in controls during first 24 hours emphasizing progressive stroke, contrary to significant improvement following NBO, tPA and $\mathrm{HBO}+\mathrm{tPA}$ treatment. Previous reports on the short-term beneficial effects of tPA $[23,28]$ are confirmed by the current data. The surprising lack of $\mathrm{HBO}$ efficacy raises several questions such as those of the pressure used (2.4 ATA), possibly suboptimal to obtain beneficial effects, but chosen due to well tolerability in patients with other diseases (e.g., problem wounds). However, previous data on hyperoxia in experimental stroke indicate efficacy predominantly in transient versus permanent ischemia $[13,17]$.
By investigating leakage phenomena on vascular structures at 2 different time points, mechanisms of ischemiainduced BBB damage were addressed quantitatively. Previous studies investigated extravasation mainly by quantifying Evans Blue [7,30,31], sodium fluorescein [16] or FITC-dextran [32] directly, or even by gadoliniumenhanced magnetic resonance imaging $[8,31,33]$. FITCalbumin has advantages as inert vehicle [26] and is comparable with FITC-dextran regarding molecular structure, which revealed significant associations to infarct size and impaired neuronal structures as shown by Fluoro-Jade staining [32]. Concerning the time course of BBB impairment basically 2 hypotheses exist: Long-lasting BBB opening (e.g., several weeks) has been reported shortly after ischemia/reperfusion [31,33], whereas other authors reported a biphasic dynamics $[7,8]$. As shown in controls, total leakage decreased from 5 to 25 hours, whereas the leakage proportion did not differ between 5 and 25 hours, 


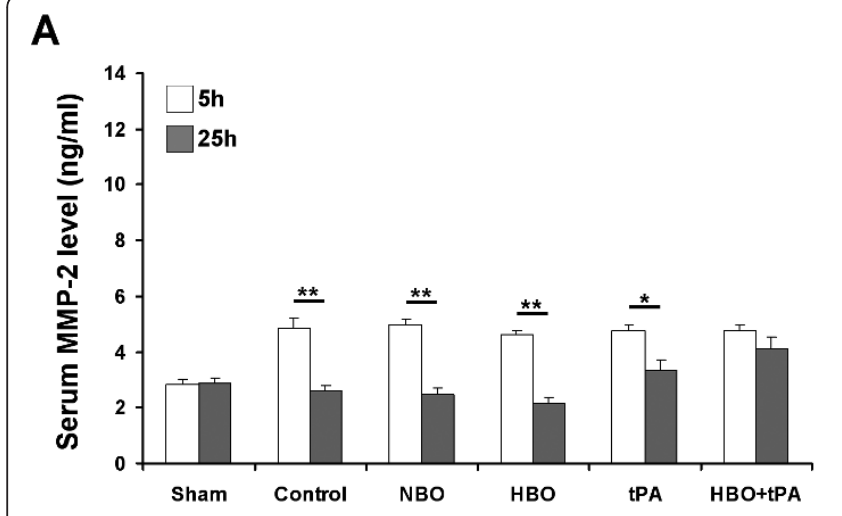

B

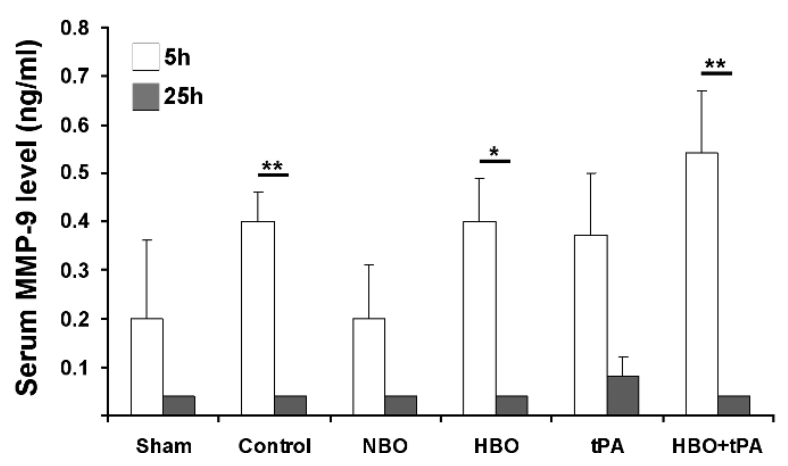

C

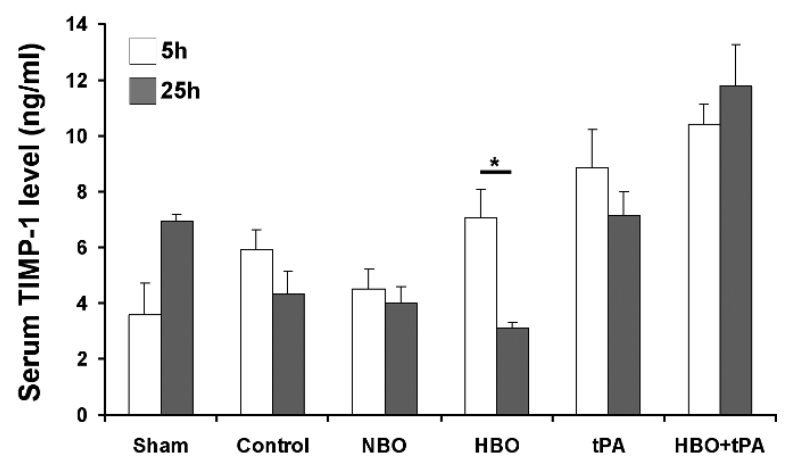

D

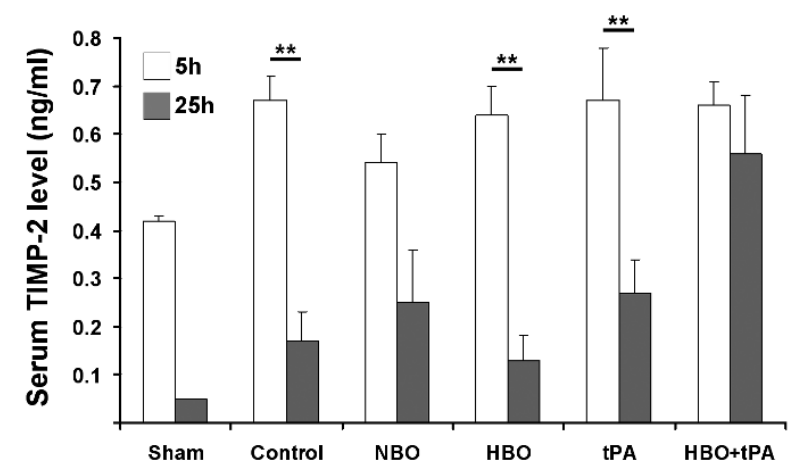

Figure 4 Serum levels of MMP-2 (A), MMP-9 (B), TIMP-1 (C), and TIMP-2 (D), obtained during final narcosis at 5 or 25 hours. Sample sizes for MMP-2, MMP-9, TIMP-1, and TIMP-2 at 5 hours (sham/control/NBO/HBO/tPA/HBO+tPA): 2/8/8/8/7/8, at 25 hours: 2/8/8/7/8/8. Bars: means, lines: standard errors, $P$-value: ${ }^{*}<0.05,{ }^{* *}<0.01$.

indicating early maximal $\mathrm{BBB}$ alteration. However, the comparison is limited by different types of ischemia, more precisely, thromboembolic in the present study versus filament-induced ischemia/reperfusion in previous reports $[7,8,31,33]$. Concerning the treatment, the present data revealed the lowest total leakage for tPA at 5 hours, most likely related to early recanalization, and tended to an improved leakage proportion for hyperoxia at 25 hours indicating BBB stabilization. As expected, use of tPA worsened $\mathrm{BBB}$ integrity at 25 hours, while co-administered HBO showed no attenuation of this effect. The loss of integrity is mainly attributed to MMP activation due to enzymatic effects of tPA $[21,34]$, as previously shown by a tPA dose-dependent correlation of BBB leakage and MMP-9 levels [20]. Interestingly, the leakage proportion solely correlated with early functional impairment, indicating that worsened $\mathrm{BBB}$ integrity following $\mathrm{TPA}$ and $\mathrm{HBO}$ + tPA were not clinically relevant at 24 hours.

MMPs as proteolytic enzymes known to degrade extracellular matrix and corresponding TIMPs with primarily inhibiting functions [9,21], were measured to reveal mechanisms of $\mathrm{BBB}$ regulation. Park et al. [35] demonstrated a significant correlation between MMP-9 values in blood and brain tissue, which enables parallel serum investigation and brain tissue analysis by immunohistochemistry. Previous studies associated vascular disruption with MMP-2 [36] and MMP-9 [30,37]. This becomes relevant in tPA-related hemorrhagic transformation [22,38], because tPA raises MMPs and TIMPs as consequence of plasminogen activation [21,34,39]. Prior studies revealed an inconsistent time course of MMPs, whereas here MMP-2 levels were found to increase within hours after ischemia mediating potentially initial BBB opening, which might be followed by a MMP-9related second opening during the first days $[9,38,40]$. Other authors noted that MMP-9 responses in the acute phase, and afterwards MMP-2 in later phases after stroke [10]. These conflicting data may result from different measurement techniques, ischemia models (transient/permanent) and animal species. Here, the MMP-9 levels at most tended to increase at 5 hours but markedly decreased at 25 hours when compared to sham, coherent with reports on later peak elevation $[9,40]$. Overall, $\mathrm{HBO}$ led to the lowest MMP-2 levels at 
25 hours (closest to NBO) and decreased MMP-9 during the first 25 hours, which is in accordance with low leakage. Veltkamp et al. [15] also demonstrated a MMP9 reduction with corresponding decreased basal lamina degradation at 24 hours following $\mathrm{HBO}$ in experimental stroke. tPA resulted in an elevated MMP-2 level, but unexpectedly HBO failed to attenuate this effect with concomitant use. The data on TIMPs resembled those of the MMPs: At 25 hours, HBO led to the lowest values and tPA-associated interventions resulted in increased TIMPs. Currently, causal links between MMPs and TIMPs remain unresolved due to different interpretations: TIMPs might be reactively elevated corresponding with MMPs, or following stroke itself even before MMPs increased, further complicated by known paradoxic (exactly not only inhibiting) roles of TIMPs [21,41]. Future stroke studies on MMP modification should consider the complexity of MMPs acute deleterious effects and their known delayed beneficial actions in recovery $[9,10]$. Zhao et al. [42] impressively demonstrated decreased infarct sizes by inhibition of MMP at day 1 , but infarct worsening by inhibition at day 7 .

The present study has some limitations: Intracerebral hemorrhage or recanalization rates were not determined, but complications were considered in mortality and functional impairment. The 24-hour observation period limits generalization and implies further research on $\mathrm{BBB}$ integrity during longer periods. Due to the existence of previous reports regarding NBO simultaneously applied to tPA [28], this combination has not been investigated, although it would be easily to implement into clinical routine. $\mathrm{HBO}$ was performed only at 2.4 ATA; a higher pressure would possibly stabilize BBB integrity, even when co-administered with tPA.

\section{Conclusions}

In summary, NBO showed promising results with decreased functional impairment and reduced BBB permeability during the first 25 hours after acute experimental stroke. Surprisingly, HBO failed to demonstrate functional improvement, but tended to stabilize BBB and to reduce MMP activation. Concomitant treatment with $\mathrm{HBO}$ and $\mathrm{TPA}$ provided early functional improvement, potentially due to enhanced recanalization, but caused increased BBB permeability and MMP-2 activation, which could impede delayed regenerative processes. Further studies are required to identify long-term interactions of tPA with both $\mathrm{NBO}$ and $\mathrm{HBO}$, especially in order to avoid unfavorable courses of combined treatment. Additionally, different duration and altered pressure of oxygen application together with tPA need to be addressed in future studies to explore potential timeand pressure-related side effects.

\section{Acknowledgements and funding}

The authors would like to thank Professor Marc Fisher (Department of Neurology, University of Massachusetts Medical School, Worcester, Massachusetts, USA) for critically revising the manuscript, and Ms. Ute Bauer (Paul Flechsig Institute for Brain Research, University of Leipzig, Leipzig, Germany) for excellent technical assistance. Dr. Petra Madaj-Sterba and Ms. Sigrid Weisheit (Medizinisch-Experimentelles Zentrum, University of Leipzig, Leipzig, Germany) are acknowledged for animal care. The Department of Neurology, University of Leipzig, Leipzig, Germany, supported this work with an internal grant.

\section{Author details}

'Department of Neurology, University of Leipzig, Liebigstr. 20, 04103 Leipzig, Germany. ${ }^{2}$ Paul Flechsig Institute for Brain Research, University of Leipzig, Jahnallee 59, 04109 Leipzig, Germany. ${ }^{3}$ Department of Anatomy, Histology and Embryology, Faculty of Veterinary Medicine, University of Leipzig, An den Tierkliniken 43, 04103 Leipzig, Germany. ${ }^{4}$ Fraunhofer Institute for Cell Therapy and Immunology, Perlickstr. 1, 04103 Leipzig, Germany.

${ }^{5}$ Translational Centre for Regenerative Medicine, University of Leipzig, Philipp-Rosenthal-Str. 55, 04103 Leipzig, Germany. ${ }^{6}$ Institute of Clinical Immunology and Transfusion Medicine, University of Leipzig, Johannisallee 30, 04103 Leipzig, Germany.

\section{Authors' contributions}

$\mathrm{DM}, \mathrm{JB}, \mathrm{CH}, \mathrm{JK}$ and $\mathrm{WH}$ conceived the present study including outcome measurements. DS facilitated funding. DM and CW conducted the animal experiments. JP and WH performed tissue preparation and serial tissue staining. JG carried out laser scanning microscopy. DM and CW recorded the functional outcome; further endpoints were assessed in a blinded manner by JP (FITC-albumin extravasation) and MK (serum MMP and TIMP evaluation). DM analyzed the data and wrote the manuscript, followed by a critical revision by WH, JB, DS and CW. All authors have read and approved the final manuscript.

\section{Competing interests}

The authors declare that they have no competing interests.

Received: 19 April 2011 Accepted: 16 June 2011

Published: 16 June 2011

\section{References}

1. Endres M, Engelhardt B, Koistinaho J, Lindvall O, Meairs S, Mohr JP, Planas A, Rothwell N, Schwaninger M, Schwab ME, Vivien D, Wieloch T, Dirnagl U: Improving outcome after stroke: overcoming the translational roadblock. Cerebrovasc Dis 2008, 25:268-278.

2. Bechmann I, Galea I, Perry VH: What is the blood-brain barrier (not)? Trends Immunol 2007, 28:5-11.

3. Kaur C, Ling EA: Blood brain barrier in hypoxic-ischemic conditions. Curr Neurovasc Res 2008, 5:71-81.

4. del Zoppo GJ: The neurovascular unit in the setting of stroke. J Intern Med 2010, 267:156-171.

5. Young AR, Ali C, Duretête A, Vivien D: Neuroprotection and stroke: time for a compromise. J Neurochem 2007, 103:1302-1309.

6. Li F, Tatlisumak T: Focal brain ischemia models in rodents. In Handbook of Experimental Neurology. Edited by: Tatlisumak T, Fisher M. Cambridge: Cambridge University Press; 2006:212-228.

7. Belayev L, Busto R, Zhao W, Ginsberg MD: Quantitative evaluation of blood-brain barrier permeability following middle cerebral artery occlusion in rats. Brain Res 1996, 739:88-96.

8. Pillai DR, Dittmar MS, Baldaranov D, Heidemann RM, Henning EC, Schuierer G, Bogdahn U, Schlachetzki F: Cerebral ischemia-reperfusion injury in rats-a $3 \mathrm{~T}$ MRI study on biphasic blood-brain barrier opening and the dynamics of edema formation. $J$ Cereb Blood Flow Metab 2009, 29:1846-1855.

9. Candelario-Jalil E, Yang Y, Rosenberg GA: Diverse roles of matrix metalloproteinases and tissue inhibitors of metalloproteinases in neuroinflammation and cerebral ischemia. Neuroscience 2009, 158:983-994.

10. Rosell A, Lo EH: Multiphasic roles for matrix metalloproteinases after stroke. Curr Opin Pharmacol 2008, 8:82-89. 
11. Schäbitz WR, Schade H, Heiland S, Kollmar R, Bardutzky J, Henninger N, Müller H, Carl U, Toyokuni S, Sommer C, Schwab S: Neuroprotection by hyperbaric oxygenation after experimental focal cerebral ischemia monitored by MRI. Stroke 2004, 35:1175-1179.

12. Liu W, Hendren J, Qin XJ, Liu KJ: Normobaric hyperoxia reduces the neurovascular complications associated with delayed tissue plasminogen activator treatment in a rat model of focal cerebral ischemia. Stroke 2009, 40:2526-2531.

13. Sun L, Zhou W, Mueller C, Sommer C, Heiland S, Bauer AT, Marti HH, Veltkamp R: Oxygen therapy reduces secondary hemorrhage after thrombolysis in thromboembolic cerebral ischemia. J Cereb Blood Flow Metab 2010, 30:1651-1660.

14. Qin Z, Karabiyikoglu M, Hua Y, Silbergleit R, He Y, Keep RF, Xi G: Hyperbaric oxygen-induced attenuation of hemorrhagic transformation after experimental focal transient cerebral ischemia. Stroke 2007, 38:1362-1367.

15. Veltkamp R, Bieber K, Wagner S, Beynon C, Siebing DA, Veltkamp C, Schwaninger M, Marti HH: Hyperbaric oxygen reduces basal lamina degradation after transient focal cerebral ischemia in rats. Brain Res 2006, 1076:231-237.

16. Veltkamp R, Siebing DA, Sun L, Heiland S, Bieber K, Marti HH, Nagel S, Schwab S, Schwaninger M: Hyperbaric oxygen reduces blood-brain barrier damage and edema after transient focal cerebral ischemia. Stroke 2005, 36:1679-1683.

17. Michalski D, Härtig W, Schneider D, Hobohm C: Use of normobaric and hyperbaric oxygen in acute focal cerebral ischemia - a preclinical and clinical review. Acta Neurol Scand 2011, 123:85-97.

18. Singhal $A B$ : A review of oxygen therapy in ischemic stroke. Neurol Res 2007, 29:173-183.

19. Hacke W, Kaste M, Bluhmki E, Brozman M, Dávalos A, Guidetti D, Larrue V, Lees KR, Medeghri Z, Machnig T, Schneider D, von Kummer R, Wahlgren N, Toni D, ECASS Investigators: Thrombolysis with alteplase 3 to 4.5 hours after acute ischemic stroke. N Engl J Med 2008, 359:1317-1329.

20. Burggraf D, Martens HK, Dichgans M, Hamann GF: rt-PA causes a dosedependent increase in the extravasation of cellular and non-cellular blood elements after focal cerebral ischemia. Brain Res 2007, 1164:55-62.

21. Adibhatla RM, Hatcher JF: Tissue plasminogen activator (tPA) and matrix metalloproteinases in the pathogenesis of stroke: therapeutic strategies. CNS Neurol Disord Drug Targets 2008, 7:243-253.

22. Jin R, Yang G, Li G: Molecular insights and therapeutic targets for bloodbrain barrier disruption in ischemic stroke: critical role of matrix metalloproteinases and tissue-type plasminogen activator. Neurobiol Dis 2010, 38:376-385.

23. Michalski D, Küppers-Tiedt L, Weise C, Laignel F, Härtig W, Raviolo M, Schneider D, Hobohm C: Long-term functional and neurological outcome after simultaneous treatment with tissue-plasminogen activator and hyperbaric oxygen in early phase of embolic stroke in rats. Brain Res 2009, 1303:161-168.

24. Zhang RL, Chopp M, Zhang ZG, Jiang Q, Ewing JR: A rat model of focal embolic cerebral ischemia. Brain Res 1997, 766:83-92.

25. Menzies SA, Hoff JT, Betz AL: Middle cerebral artery occlusion in rats: a neurological and pathological evaluation of a reproducible model. Neurosurgery 1992, 31:100-106.

26. Michalski D, Grosche J, Pelz J, Schneider D, Weise C, Bauer U, Kacza J, Gärtner U, Hobohm C, Härtig W: A novel quantification of blood-brain barrier damage and histochemical typing after embolic stroke in rats. Brain Res 2010, 1359:186-200

27. Sternberger $\mathrm{NH}$, Sternberger LA: Blood-brain barrier protein recognized by monoclonal antibody. Proc Natl Acad Sci USA 1987, 84:8169-8173.

28. Fujiwara N, Murata Y, Arai K, Egi Y, Lu J, Wu O, Singhal AB, Lo EH: Combination therapy with normobaric oxygen (NBO) plus thrombolysis in experimental ischemic stroke. BMC Neurosci 2009, 10:79.

29. Henninger N, Bratane BT, Bastan B, Bouley J, Fisher M: Normobaric hyperoxia and delayed tPA treatment in a rat embolic stroke model. J Cereb Blood Flow Metab 2009, 29:119-129.

30. Gasche Y, Fujimura M, Morita-Fujimura Y, Copin JC, Kawase M, Massengale J, Chan PH: Early appearance of activated matrix metalloproteinase- 9 after focal cerebral ischemia in mice: a possible role in blood-brain barrier dysfunction. J Cereb Blood Flow Metab 1999, 19:1020-1028.

31. Strbian D, Durukan A, Pitkonen M, Marinkovic I, Tatlisumak E, Pedrono E, Abo-Ramadan U, Tatlisumak T: The blood-brain barrier is continuously open for several weeks following transient focal cerebral ischemia. Neuroscience 2008, 153:175-181.

32. Chen B, Friedman B, Cheng Q, Tsai P, Schim E, Kleinfeld D, Lyden PD: Severe blood-brain barrier disruption and surrounding tissue injury. Stroke 2009, 40:e666-e674.

33. Abo-Ramadan U, Durukan A, Pitkonen M, Marinkovic I, Tatlisumak E, Pedrono E, Soinne L, Strbian D, Tatlisumak T: Post-ischemic leakiness of the blood-brain barrier: a quantitative and systematic assessment by Patlak plots. Exp Neurol 2009, 219:328-333.

34. Kelly MA, Shuaib A, Todd KG: Matrix metalloproteinase activation and blood-brain barrier breakdown following thrombolysis. Exp Neurol 2006, 200:38-49.

35. Park KP, Rosell A, Foerch C, Xing C, Kim WJ, Lee S, Opdenakker G, Furie KL, Lo EH: Plasma and brain matrix metalloproteinase- 9 after acute focal cerebral ischemia in rats. Stroke 2009, 40:2836-2842.

36. Fukuda S, Fini CA, Mabuchi T, Koziol JA, Eggleston LL Jr, del Zoppo GJ: Focal cerebral ischemia induces active proteases that degrade microvascular matrix. Stroke 2004, 35:998-1004.

37. Bauer AT, Bürgers HF, Rabie T, Marti HH: Matrix metalloproteinase-9 mediates hypoxia-induced vascular leakage in the brain via tight junction rearrangement. J Cereb Blood Flow Metab 2010, 30:837-848.

38. Heo JH, Lucero J, Abumiya T, Koziol JA, Copeland BR, del Zoppo GJ: Matrix metalloproteinases increase very early during experimental focal cerebral ischemia. J Cereb Blood Flow Metab 1999, 19:624-633.

39. Tsuji K, Aoki T, Tejima E, Arai K, Lee SR, Atochin DN, Huang PL, Wang X, Montaner J, Lo EH: Tissue plasminogen activator promotes matrix metalloproteinase- 9 upregulation after focal cerebral ischemia. Stroke 2005, 36:1954-1959.

40. Planas AM, Solé $S$, Justicia C: Expression and activation of matrix metalloproteinase- 2 and -9 in rat brain after transient focal cerebral ischemia. Neurobiol Dis 2001, 8:834-846.

41. Ethell IM, Ethell DW: Matrix metalloproteinases in brain development and remodeling: synaptic functions and targets. J Neurosci Res 2007, 85:2813-2823.

42. Zhao BQ, Wang S, Kim HY, Storrie H, Rosen BR, Mooney DJ, Wang X, Lo EH: Role of matrix metalloproteinases in delayed cortical responses after stroke. Nat Med 2006, 12:441-445.

doi:10.1186/2040-7378-3-5

Cite this article as: Michalski et al:: Early outcome and blood-brain barrier integrity after co-administered thrombolysis and hyperbaric oxygenation in experimental stroke. Experimental \& Translational Stroke Medicine 2011 3:5.

\section{Submit your next manuscript to BioMed Central and take full advantage of:}

- Convenient online submission

- Thorough peer review

- No space constraints or color figure charges

- Immediate publication on acceptance

- Inclusion in PubMed, CAS, Scopus and Google Scholar

- Research which is freely available for redistribution

Submit your manuscript at www.biomedcentral.com/submit
C Biomed Central 